\title{
Non-Protein-Bound Iron Is Elevated in Cerebrospinal Fluid from Preterm Infants with Posthemorrhagic Ventricular Dilatation
}

\author{
KARIN SAVMAN, ULF A. NILSSON, MATS BLENNOW, INGEMAR KJELLMER, AND \\ ANDREW WHITELAW \\ Departments of Pediatrics, Göteborg University, The Queen Silvia Children's Hospital, S-416 85 \\ Göteborg, Sweden [K.S., I.K.]; Department of Physiology, Göteborg University, S-40530 Göteborg \\ Sweden; Neonatal Unit K78, Children's Hospital, Huddinge University Hospital, S-141 86 Huddinge, \\ Sweden [M.B.]; and Division of Child Health, University of Bristol Medical School, Southmead Hospital, \\ Bristol BS10 5NB, United Kingdom [A.W.].
}

\begin{abstract}
Posthemorrhagic ventricular dilatation (PHVD) is closely associated with white matter injury and neurologic disability in the preterm infant. An important factor in periventricular white matter damage may be the specific vulnerability of iron-rich immature oligodendroglia to reactive oxygen species toxicity. Non-protein-bound iron (NPBI) is a potent catalyst in the generation of hydroxyl radicals (Fenton reaction). Our objective was to determine whether NPBI is increased in cerebrospinal fluid (CSF) from preterm infants with PHVD compared with preterm control infants. Samples of CSF were obtained from 20 infants with PHVD and 10 control subjects. The level of NPBI was determined by a new spectrophotometric method using bathophenanthroline as a chelator. To evaluate the effect of hemolysis, CSF and blood were mixed in different proportions, spun, frozen and thawed, and then analyzed for NPBI. NPBI was found in $75 \%$ (15 of 20) of infants with PHVD and in $0 \%$ (0 of 10$)$ of control infants $(p=0.0002)$. Hemolysis induced in vitro did not
\end{abstract}

\section{ABSTRACT}

result in any significant levels of NPBI. Within the group with PHVD, NPBI concentrations in CSF did not correlate with disability, parenchymal brain lesions, or the need for shunt surgery. NPBI was increased in CSF from preterm infants with PHVD, and the increase could not be explained by hemolysis alone. Free iron may help to explain the association between intraventricular hemorrhage and white matter damage. (Pediatr Res 49: 208-212, 2001)
Abbreviations:
BPS, bathophenanthroline disulfonate
CSF, cerebrospinal fluid
IVH, intraventricular hemorrhage
NPBI, non-protein-bound iron
PHVD, posthemorrhagic ventricular dilatation
ROS, reactive oxygen species

Periventricular white matter damage is the main cause of permanent neurologic impairment in the preterm infant $(1,2)$. It has also been demonstrated that IVH, particularly in association with ventriculomegaly, is strongly correlated with the subsequent development of white matter damage (3). At least two possible, but not mutually exclusive, etiologies for white matter damage are widely accepted: ischemia-reperfusion injury in an area with specific vascular characteristics (4-9) and proinflammatory cytokines released in response to infection (e.g. chorioamnionitis) or hypoxia-ischemia (10-13). The specific mechanisms triggered by these factors are not fully understood, but it has been suggested that immature iron-rich

Received May 10, 2000; accepted August 18, 2000.

Correspondence and reprint requests: Karin Savman, M.D., Department of Pediatrics, The Queen Silvia Children's Hospital, S-416 85 Göteborg, Sweden.

Supported by the Medical Research Council (grant 2591), Sweden, and the Ax:sonJohnson Foundation. oligodendroglia may be the primary target cells for periventricular white matter injury and that ROS toxicity is important in this process (14). Immature oligodendroglia express an intrinsic vulnerability to ROS-mediated injury in vitro, and free radical scavengers protect immature oligodendroglia from injury $(15,16)$. Cerebral ischemia in fetal sheep induces production of ROS (17), and asphyxia in fetal lambs showed high levels of ROS associated with white matter damage (18). The hydroxyl radical is a highly reactive oxygen species that initiates lipid peroxidation as well as direct DNA damage (19). The hydroxyl radical is generated from hydrogen peroxide, a reaction that requires a transitional metal, most importantly NPBI (via Fenton reaction) (20). The iron-chelator deferoxamine reduces brain injury after cerebral ischemia in the neonatal rat as well as after hypoxia-ischemia in newborn lambs $(21,22)$. In cell cultures deferoxamine prevents injury to oligodendroglia in a similar way as free radical scavengers $(16$, 
23). In spite of experimental studies supporting a role for iron in neonatal brain damage, the link between white matter damage in the preterm infant and iron-induced ROS activation is mostly based on studies in cell cultures. In term infants with asphyxia and adverse outcome, the level of NPBI in plasma is elevated (24), but to our knowledge there are no clinical studies on NPBI in relation to white matter injury in the preterm infant.

In this study we use a new method for determining NPBI that specifically measures the level of non-protein-bound ferrous iron, the reactive form of NPBI that directly acts as a catalyst in the formation of hydroxyl radicals. In addition, all NPBI in CSF is likely to be in its reduced ferrous state (25), and the level of ferrous iron will directly reflect the total amount of NPBI.

The purpose of this study was to determine whether NPBI is increased in CSF from preterm infants with PHVD and subsequent high risk of developing white matter damage compared with preterm control infants.

\section{METHODS}

Patients. Twenty infants with gestational age $\leq 36$ wk with IVH and PHVD were included in the study. Infants with systemic infection with positive cultures from blood or CSF were excluded. The diagnoses IVH, PHVD, and associated white matter lesions were established by repeated ultrasonography of the brain. PHVD was diagnosed when IVH was followed by a progressive increase in ventricular width to 4 $\mathrm{mm}$ over the 97th centile (26). Dilatation driven by CSF under pressure was differentiated from dilatation caused by atrophy of periventricular tissue by 1) ballooning of the lateral and third ventricles, 2) normal or accelerated head expansion over time, and 3) CSF pressure $>6 \mathrm{~mm} \mathrm{Hg}$ (27). Parenchymal brain lesions in the white matter were identified as 1) parenchymal echodensities in continuity with the ventricle which subsequently become echolucent, or 2) persistent periventricular echodensities or echolucencies separate from the ventricular margin. The surgical insertion of a ventriculoperitoneal shunt was recorded. All surviving infants were followed up for 12 mo to $8 \mathrm{y}$, and the neurologic outcome was divided into 1 ) within normal limits for age, 2) motor disability alone, or 3 ) multiple disability. The control group consisted of 10 infants with gestational age $\leq 36 \mathrm{wk}$ with clinical signs of infection but with negative cultures from blood and CSF and no findings suggesting CNS pathologic disease. The infants were treated in the neonatal intensive care units at Aker Hospital, Oslo; Southmead Hospital, Bristol; Huddinge University Hospital, Stockholm; and The Queen Silvia Children's Hospital, Göteborg.

CSF sampling. CSF was obtained by spinal or ventricular taps performed for diagnostic or therapeutic reasons. After samples for diagnostic purposes had been obtained, an extra volume of $\leq 1 \mathrm{~mL} / \mathrm{kg}$ was collected. In the case of therapeutic taps, larger volumes were obtained. The samples were spun at $4000 \mathrm{rpm}$ for $10 \mathrm{~min}$, and the supernatant was frozen at $-70^{\circ} \mathrm{C}$. Parental consent was obtained for the extra amount of CSF taken, and the study was approved by the research ethics committee of each hospital.
In vitro hemolysis. Blood from an adult who had taken iron supplementation for $3 \mathrm{wk}$ was mixed with CSF from a preterm infant (gestational age $32 \mathrm{wk}$ ) with cerebral malformation and normal cell and protein counts. Two different proportions were used for blood in CSF, 1:50 and 1:100. The background absorption of these samples was similar to that of the samples collected from the study infants. The mixed samples as well as CSF without blood were incubated at $37^{\circ} \mathrm{C}$ for up to $1 \mathrm{wk}$. At days $0,1,3$, and 7 , samples were analyzed for NPBI. The samples were frozen and thawed; spun, frozen, and thawed; or frozen, thawed, and spun before analysis. Hemolysis was induced in several samples after $3 \mathrm{~d}$ and in all samples after $7 \mathrm{~d}$ of incubation.

NPBI analysis. NPBI was analyzed by a new method using bathophenanthroline disulfonate to chelate ferrous iron, thus forming a complex that could be analyzed by spectrophotometry. The standard curves are linear up to concentrations of 50 $\mu \mathrm{M}$ and are similar in plasma, CSF, and water, which allows the absorbance in CSF samples to be quantified against standards conveniently made up in water as described below. The presence of zinc or copper does not interfere with the measurements of iron. The detection level is $0.5 \mu \mathrm{M}$ (UA Nilsson, M Bassen, K Savman, I Kjellmer, manuscript submitted).

Chemicals. BPS (4,7-diphenyl-1,10-phenanthroline disulfonate) and ferrous ammonium sulfate $\left[\left(\mathrm{NH}_{4}\right)_{2} \mathrm{Fe}\left(\mathrm{SO}_{4}\right)_{2}\right]$ of the highest purity commercially available were purchased from local suppliers (Sigma-Aldrich Chemical Co., Stockholm Sweden).

Materials. Samples were filtered using the Millipore MultiScreen Filtration System (MAHV N45 10), including MultiScreen Vacuum Manifold and MultiScreen-HV clear plates with $0.45 \mu \mathrm{m}$ hydrophilic low protein binding Durapore membrane and lid. After filtration the samples were pipetted into polystyrene $1 / 2$ area 96 -well plates (Costar 3690). Absorbances were read with a plate reader from Molecular Devices (SPECTRA max plus) operating under Softmax Pro 2.4 software.

Standard curves. A $50 \mathrm{mM}$ stock solution of the ferrous iron chelator BPS was prepared by dissolving $28.2 \mathrm{mg}$ BPS in 1000 $\mu \mathrm{L}$ of deionized pyrogen-free water. A standard curve was constructed by dissolving $196 \mathrm{mg}$ of ferrous ammonium sulfate in $500 \mathrm{~mL}$ of deionized pyrogen-free water, giving a $1 \mathrm{mM}$ stock solution of ferrous ion. This was further diluted with water to produce solutions of $0.1 \mu \mathrm{M}$ to $50 \mu \mathrm{M}$. Then $10 \mu \mathrm{L}$ of the BPS stock was added to $490 \mu \mathrm{L}$ of each of these solutions. After mixing, the samples were left for $15 \mathrm{~min}$, and then $50 \mu \mathrm{L}$ of each sample was transferred to a $96-$ well plate. Finally, absorbance was read in the plate reader at $535 \mathrm{~nm}$ against a water blank.

Measurements in CSF. Forty-nine microliters of CSF was pipetted into wells, and absorbance was read at $535 \mathrm{~nm}$ to give the blank absorbance for each sample. Then $1 \mu \mathrm{L}$ of the BPS stock solution was pipetted into each well. After mixing, the plate was left for $15 \mathrm{~min}$, and the absorbance was again read at $535 \mathrm{~nm}$. After subtraction of the blank values, the readings were quantified against the standard curve

Statistical analyses. All results are given as medians and interquartile range unless stated otherwise. Fisher's exact test was used to test the difference in NPBI occurrence between 
groups. The Kruskall-Wallis test and the Mann-Whitney U test were used to test differences in iron level between groups with different outcome or need for shunting. The Spearman rank test was used to test correlation between age at lumbar puncture and NPBI level.

\section{RESULTS}

Group data on the PHVD group and the control group are given in Table 1. Thirteen of 20 infants with PHVD had white matter lesions on ultrasound. The outcome in the PHVD group was normal in 6 of 20 infants, motor disability was found in 8 of 20 infants, and severe multiple disability in 6 of 20 infants. Three infants, all with severe brain damage and obvious multiple disability, died before 12 mo. In the PHVD group 6 of 17 surviving infants developed permanent hydrocephalus requiring ventriculoperitoneal shunting, while the PHVD resolved spontaneously in the rest of the surviving infants. The outcome was normal in all control infants.

NPBI was present in CSF in 75\% (15 of 20) of infants with PHVD and $0 \%$ ( 0 of 10$)$ of control infants $(p=0.0002$, Fisher's exact test). The individual values are shown in Figure 1. There was no difference in NPBI levels between infants with or without need for shunting (Mann-Whitney U test), between infants with or without white matter lesions (Mann-Whitney U test), or between infants with normal outcome, motor disability, or multiple disability (Kruskall-Wallis test).

Hemolysis induced in vitro by incubation or by freezing and thawing did not result in any significant levels of NPBI.

There was no correlation between the age at lumbar puncture and the NPBI level within the PHVD group (Spearman rank test).

\section{DISCUSSION}

NPBI was found frequently and at high levels in CSF from preterm infants with PHVD and associated white matter damage. No iron was found in CSF from preterm control infants.

Iron is the most abundant transitional metal in the brain. Studies in neonatal rats show that the uptake and concentration of iron is high in the developing brain $(28,29)$. Ferritin-bound iron can be found in white matter of the human brain in the second trimester (30), and iron is later required for active myelination (31). In spite of its crucial importance to normal metabolism and development of the brain, NPBI is potentially harmful through its capacity to generate hydroxyl radicals and induce oxidative damage. Iron in the brain is thus normally sequestered within storage proteins such as ferritin, and in CSF and plasma it is bound to transferrin (32). Ferritin synthesis in oligodendroglial cell cultures is induced by hypoxia, and fer-

Table 1. Clinical data on the studied infants

\begin{tabular}{lcccc}
\hline \multicolumn{1}{c}{ Group } & GA (wk) & Birth weight $(\mathrm{g})$ & Age at LP (d) & Sex $(\mathrm{M} / \mathrm{F})$ \\
\hline PHVD $(n=20)$ & $27(26-29)$ & $1015(805-1480)$ & $19(15-23)$ & $11 / 9$ \\
Controls $(n=10)$ & $29(27-30)$ & $1207(1062-1485)$ & $16(4-21)$ & $8 / 2$ \\
& NS & NS & $p=0.02$ & NS \\
\hline
\end{tabular}

Values as median (interquartile range). For statistical analysis MannWhitney U test and Fisher's exact test were used. GA = gestational age, LP = lumbar puncture, $\mathrm{NS}=$ not significant.

\section{NPBI $\mu \mathrm{mol} / \mathrm{L}$}

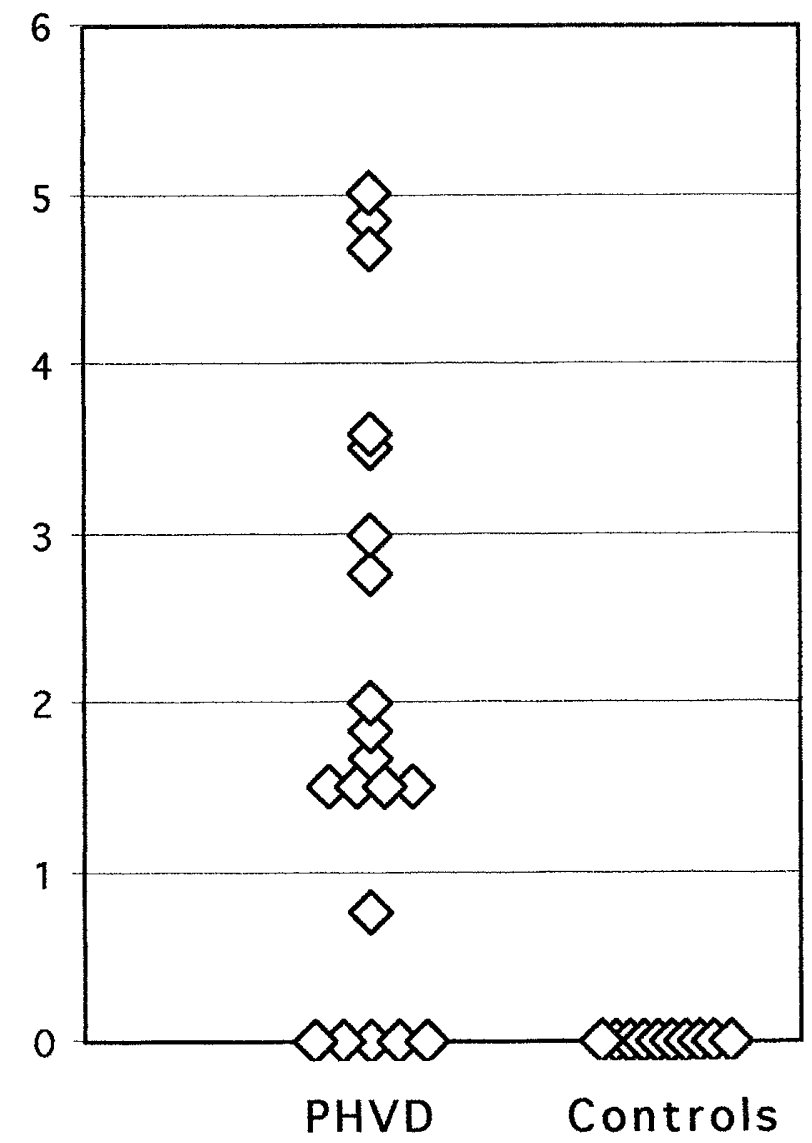

Fig. 1. Individual levels of NPBI ( $\mu \mathrm{mol} / \mathrm{L})$ in CSF from preterm infants with $\operatorname{PHVD}(n=20)$ and from preterm control infants $(n=10)$ are shown.

ritin synthesis can be increased in oligodendroglial cells by addition of exogenous iron or hydrogen peroxide. Furthermore, ferritin synthesis can be blocked by iron chelators or base (33, 34). Thus ferritin appears to be a protective mechanism against oxidative damage after hypoxia or acidosis. Our findings of high levels of NPBI in CSF suggest that iron is either released in excess or that the ability to bind and inactivate NPBI is inadequate in the preterm infant with PHVD.

There are several possible sources of NPBI including release of iron from ferritin and ferrous iron liberated during heme catabolism. Several conditions associated with hypoxiaischemia and subsequent ROS activation have the capacity to release iron from ferritin including acidosis (35) and superoxide radicals (36). A recent study in neonatal rats demonstrates that iron reaction product accumulates in injured neurons as early as $4 \mathrm{~h}$ after hypoxia-ischemia, indicating a relocation of iron within the brain (37). High concentrations of NPBI in CSF would require release from ferritin as well as leakage from iron-containing cells. In a previous study of infants with PHVD, high levels of brain-specific proteins (NFP, S-100, and GFAP) were found in CSF, indicating cellular injury and protein-leakage from neurons as well as glial cells (38). It is 
likely that leakage of iron could also occur under these circumstances.

Iron released during heme catabolism is another possible source of NPBI in CSF, especially because degrading blood is already present in the ventricular system of infants with PHVD. Hb can without further degradation potentiate excitotoxic injury in neuronal cultures, and the neurotoxic effect is attenuated by free radical scavengers as well as by deferoxamine (39), implicating iron and ROS in $\mathrm{Hb}$ toxicity. Lipid peroxidation is induced in rat brain by injections of $\mathrm{Hb}$ but only if iron is present in the protoporphyrin ring (40). The degradation of heme and release of iron is catalyzed by heme oxygenase, an enzyme induced in brain after ischemia as well as after intracerebral hemorrhage in rat $(41,42)$. Heme-induced oxidative damage in cell cultures stimulates the production of both heme oxygenase and ferritin (43). Ferritin has a very strong cytoprotective effect through its capacity to sequester iron (43), suggesting that the breakdown of $\mathrm{Hb}$ will also result in protective measures to prevent oxidative damage.

In this study we performed in vitro experiments on hemolysis in a mixture of adult blood and preterm CSF subjected to incubation, freezing, and thawing without being able to detect any significant amounts of NPBI. This could serve as a check on our methodology, confirming that handling of samples containing heme iron did not result in the release of NPBI, but it could not be used to demonstrate that such a release does not take place in vivo. The degradation of heme requires activated cells as well as an intact enzyme system (44), and it is also possible that the binding capacity for iron in adult blood exceeds that of the preterm infant (45).

There are several studies that point to the fact that the iron-binding capacity is low in the newborn infant. The ironbinding capacity in plasma is lower and the level of NBPI is higher in neonates compared with adults $(45,46)$. Cord blood plasma has a limited capacity to prevent iron-induced lipid peroxidation and may instead stimulate peroxidation (45), and the iron-binding capacity is lower in preterm infants with IVH than control infants (47). CSF in itself has also a low binding capacity that is close to saturation under normal conditions (32).

In our study we found no correlation between the absolute level of NPBI and outcome or between NPBI and the degree of white matter damage. Such a correlation would have strengthened the connection between NPBI and white matter damage if NPBI is toxic to white matter in a linear dose-dependent manner. NPBI is, however, likely to exert its harmful effect as a catalyst in hydroxyl formation, a process in which ferrous ions are turned into ferric. This process is not likely to be classically dose dependent. CSF, at least from adults, has also a very high ratio of ascorbate to ceruloplasmin. Any iron in CSF that is not bound to transferrin is therefore likely to be reduced to its active ferrous form until all ascorbate is consumed (25).

The timing of the sampling is another factor that may influence the level of NPBI and its correlation to outcome. Serial ultrasounds have demonstrated that $90 \%$ of IVH in preterm infants occur within the first $3 \mathrm{~d}$ after birth (4). The infant's age at sampling will thus roughly reflect the duration of the bleeding. We found no correlation between the age at lumbar puncture and the level of NPBI, but only frequent serial measurements - not performed in this study - would give information on the time course of NPBI levels and any connection between peak levels and outcome.

In conclusion, our study demonstrated that NPBI was found frequently and at high levels in CSF from preterm infants with PHVD, the majority of who had white matter lesions and subsequent disability. Irrespective of its origin, NPBI has the capacity to initiate and maintain oxidative stress and may contribute to white matter damage as well as provide a link between IVH and white matter damage.

\section{REFERENCES}

1. Pinto-Martin J, Riolo S, Cnaan A, Holzman C, Susser M, Paneth N 1995 Cranial ultrasound prediction of disabling and nondisabling cerebral palsy at two in a low birth weight population. Pediatrics 95:249-254

2. Pinto-Martin J, Whitaker A, Feldman J, Rossem RV, Paneth N 1999 Relation of cranial ultrasound abnormalities in low-birth weight infants to motor or cognitive performance at ages 2, 6 and 9 years. Dev Med Child Neurol 41:826-833

3. Kuban K, Sanocka U, Leviton A, Allred EN, Pagano M, Dammann O, Share J, Rosenfeld D, Abiri M, DiSalvo D, Doubilet P, Kairam R, Kazam E, Kirpekar M, Schonfeld S 1999 White matter disorders of prematurity: association with intraventricular hemorrhage and ventriculomegaly. J Pediatr 134:539-546

4. Volpe J 1995 Neurology of the Newborn, 3rd Ed. WB Saunders, Philadelphia, pp 403-463

5. Larroche JC 1984 Perinatal brain damage. In: Adams JH, Corselli J, Duchen LW (eds) Greenfield's Neuropathology. Edward Arnold, London, pp 451-489

6. Young S, Hernandez M, Yagel S 1982 Selective reduction in blood flow to white matter during hypotension in newborn dogs: a possible mechanism of periventricular leukomalacia. Ann Neurol 12:445-448

7. Miyawaki T, Matsui K, Takashima S 1998 Developmental characteristics of vessel density in the human fetal and infant brains. Early Hum Dev 53:65-72

8. Pryds O, Greisen G, Lou H, Friis-Hansen B 1989 Heterogeneity of cerebral vasoreactivity in preterm infants supported on mechanical ventilation. J Pediatr 115:638645

9. Borch K, Greisen G 1998 Blood flow distribution in the normal human preterm brain. Pediatr Res 43:28-33

10. Leviton A 1993 Preterm birth and cerebral palsy: is tumor necrosis factor the missing link? Dev Med Child Neurol 35:549-558

11. Damman O, Leviton A 1998 Infection remote from the brain, neonatal white matter injury and cerebral palsy in the preterm infant. Semin Pediatr Neurol 5:190-201

12. Yoon B, Romero R, Yang S, Jun J, Kim I, Choi J 1996 Interleukin-6 concentrations in umbilical cord plasma are elevated in neonates with white matter lesions associated periventricular leukomalacia. Am J Obstet Gynecol 174:1433-1440

13. Hagberg H, Gilland E, Bona E, Hansson L-Å, Hahn-Zoric M, Blennow M, Holst M, McRae A, Söder O 1996 Enhanced expression of interleukin (IL)-1 and IL-6 messenger RNA and bioactive protein after hypoxia-ischemia in neonatal rats. Pediatr Res 40:603-609

14. Back S, Volpe J 1997 Cellular and molecular pathogenesis of periventricular white matter damage. Ment Retard Dev Disabil Res Rev 3:96-107

15. Oka A, Belliveau M, Rosenberg P, Volpe J 1993 Vulnerability of oligodendroglia to glutamate: pharmacology, mechanisms and prevention. J Neurosci 13:1441-1453

16. Yonesawa M, Back S, Gan X, Rosenberg P, Volpe J 1996 Cystine deprivation induces oligodendroglial death: rescue by free radical scavengers and by a diffusible glial factor. J Neurochem 67:566-573

17. Bågenholm R, Nilsson U, Gotborg C, Kjellmer I 1998 Free radicals are formed in the brain of fetal sheep during reperfusion after cerebral ischemia. Pediatr Res 43:271275

18. Ikeda T, Choi B, Yee S, Murata Y, Quilligan E 1999 Oxidative stress, brain white matter damage and intrauterine asphyxia in fetal lambs. Int J Dev Neurosci 17:1-14

19. Halliwell B, Aruoma O 1991 DNA damage by oxygen-derived species: its mechanism and measurement in mammalian systems. FEBS 281:9-19

20. Halliwell B, Gutteridge J, Cross C 1992 Free radicals, antioxidants and human disease: where are we now? J Lab Clin Med 119:598-620

21. Palmer C, Roberts R, Bero C 1994 Deferoxamine posttreatment reduces ischemic brain injury in neonatal rats. Stroke 25:1039-1045

22. Shadid M, Buoncore G, Groenendaal F, Moison R, Ferrali M, Berger HM, van Bel F 1998 Effect of deferoxamine and allopurinol on non-protein-bound iron concentrations in plasma and cortical brain tissue of newborn lambs following hypoxiaischemia. Neurosci Lett 248:5-8

23. Thorburne S, Juurlink B 1996 Low glutathione and high iron govern the susceptibility of oligodendroglial precursors to oxidative stress. J Neurochem 67:1014-1022

24. Dorrepaal C, Berger H, Benders M, Zoeren-Grobben DV, Bor MVD, Bel FV 1996 Nonprotein-bound iron in postasphyxial reperfusion injury in the newborn. Pediatrics 98:883-889

25. Gutteridge J 1992 Ferrous iron is detected in cerebrospinal fluid using bleomycin and DNA damage Clin Sci 82:315-320 
26. Levene M 1981 Measurement of the growth of the lateral ventricles in preterm infants with real time ultrasound Arch Dis Child 56:900-904

27. Whitelaw A 1995 Neonatal hydrocephalus. In: Levene M, Lilford R (eds) Fetal and Neonatal Neurology and Neurosurgery. Churchill Livingstone, Edinburgh, pp 645 659

28. Taylor E, Morgan E 1990 Developmental changes in transferrin and iron uptake by the brain in the rat. Brain Res Dev Brain Res 55:35-42

29. Roskams A, Connors J 1994 Iron, transferrin and ferritin in the rat brain during development and aging. J Neurochem 63:709-716

30. Ozawa H, Nishida A, Mito T, Takashima S 1994 Development of ferritin-containing cells in the pons and cerebellum of the human brain. Brain Dev 16:92-95

31. Connor J, Menzies S 1996 Relationship of iron to oligodendrocytes and myelination. Glia 17:83-93

32. Gutteridge J 1992 Iron and oxygen radicals in brain. Ann Neurol 32(suppl):S16-S21

33. Qi Y, Dawson G 1994 Hypoxia specifically and reversibly induces the synthesis of ferritin in oligodendrocytes and human oligodendrogliomas. J Neurochem 63:1485-1490

34. Qi J, Jamindar T, Dawson G 1995 Hypoxia alters iron homeostasis and induces ferritin synthesis in oligodendrocytes. J Neurochem 64:2458-2464

35. Ying W, Han S, Miller J, Swanson R 1999 Acidosis potentiates oxidative neuronal death by multiple mechanisms. J Neurochem 73:1549-1556

36. Bolann B, Ulvik R 1987 Release of iron from ferritin by xanthine oxidase: role of the superoxide radical. Biochem J 243:55-59

37. Palmer C, Menzies S, Roberts R, Pavlick G, Connor J 1999 Changes in iron histochemistry after hypoxic-ischemic brain injury in the neonatal rat. Neurosci Res 56:60-71
38. Whitelaw A, Rosengren L, Blennow M 2000 Brain specific proteins in CSF after intraventricular hemorrhage. Arch Dis Child (in press)

39. Regan R, Panter S 1996 Hemoglobin potentiates excitotoxic injury in cortical cell culture. J Neurotrauma 13:223-231

40. Willmore L, Triggs W 1991 Iron-induced lipid peroxidation and brain injury responses. Int J Dev Neurosci 9:175-180

41. Paschen W, Uto A, Djuricic B, Schmitt J 1994 Hemeoxygenase expression after reversible ischemia of rat brain. Neurosci Lett 180:5-8

42. Matz P, Weinstein P, Sharp F 1997 Heme oxygenase-1 and heat shock protein 70 induction in glia and neurons throughout rat brain after experimental intracerebral hemorrhage. Neurosurgery 40:152-160

43. Balla G, Jacob H, Balla J, Rosenberg M, Nath K, Apple F, Eaton JW, Vercellotti GM 1992 Ferritin: a cytoprotective antioxidant stratagem of endothelium. J Biol Chem 267:18148-18153

44. Koeppen A, Dickson A, McEvoy J 1995 The cellular reactions to experimental intracerebral hemorrhage. J Neurol Sci 134(suppl):102-112

45. Lindeman J, Houdkamp E, Lentjes E, Poorthuis B, Berger H 1992 Limited protection against iron-induced lipid peroxidation by cord blood plasma. Free Radic Res Commun 16:285-294

46. Evans P, Evans R, Kovar I, Holton A, Halliwell B 1992 Bleomycin-detectable iron in the plasma of premature and full-term neonates. FEBS Lett 303:210-212

47. Lackmann G, Hesse L, Tollner U 1996 Reduced iron-associated anti-oxidants in premature newborns suffering intracerebral hemorrhage. Free Radic Biol Med 20:407-409 